\title{
The Man in the High Castle de Philip K. Dick : et si on changeait le contexte?
}

\section{Sandrine Sorlin}

\section{(2) OpenEdition \\ 1 Journals}

Édition électronique

URL : http://journals.openedition.org/esa/1596

DOI : 10.4000/esa.1596

ISSN : 2650-2623

Éditeur

Société de stylistique anglaise

Édition imprimée

Date de publication : 31 décembre 2011

Pagination : $31-43$

ISSN : 2116-1747

\section{Référence électronique}

Sandrine Sorlin, «The Man in the High Castle de Philip K. Dick : et si on changeait le contexte? », Études

de stylistique anglaise [En ligne], 3 | 2011, mis en ligne le 27 novembre 2018, consulté le 23 avril 2019.

URL : http://journals.openedition.org/esa/1596 ; DOI : 10.4000/esa.1596 


\title{
THE MAN IN THE HIGH CASTLE DE PHILIP K. DICK : ET SI ON CHANGEAIT LE CONTEXTE?
}

\author{
Sandrine Sorlin \\ Université Paul Valery-Montpellier 3, EMMA
}

\begin{abstract}
At the end of WWII, in an inverted historical context - the two victorious empires, Germany and Japan, have carved the planet among themselves-, the world is cut up along racial and highly hierarchical social lines that compel people to find their 'place' even if it means undergoing linguistic alienation. Conversations have turned into pitched battles where each linguistic move can be a humiliating flop or a victorious advance over the other. Authentic thoughts are restricted to the narration-a space of incommunicability where the borders between the different types of discourse have become blurred. Human beings are in fact more « spoken » by an ideological rhetoric than they really «speak» (to one another). Indeed The Man in the High Castle shows the frightful effects of a propaganda that has succeeded since it is no longer perceived as manipulation. Besides, communication is all the more problematic as it is subtended by two cultural (oriental / occidental) codes that are widely incompatible. If the Germans yearn after a borderless world pertaining to the linguistic «notion», that is completely out of context and unconcerned with individuality, the Japanese are supported by an ancestral book, the $I$ Ching, that privileges the immeasurably small in permanent, ever renewed contextualisation. In the new geopolitical context depicted by Philip K. Dick, the change in protagonists has changed nothing to the dramatic scene: the modes of exploitation and colonisation seem to be indifferent to context.
\end{abstract}

Keywords: dystopia, contextualisation, pragmatics, rhetoric, propaganda, xenophobia, metaphor

Cet article, accepté par le comité de lecture, est issu de la communication présentée en mai 2011 dans l'atelier de stylistique lors du $51^{\text {ème }}$ Congrès de la SAES à Paris Diderot et Paris Sorbonne Nouvelle. 


\section{Introduction}

Dans The Man in the High Castle, Philip K. Dick imagine ce que serait le monde si l'axe germano-japonais était sorti vainqueur de la seconde guerre mondiale. C'est le refus des États-Unis de s'engager dans le conflit mondial auprès des forces alliées contre l'Allemagne nazie - au nom d'une politique isolationniste visant à sortir le pays de la grande dépression - qui explique le nouveau contexte géopolitique : sans frein pour l'arrêter, l'Allemagne nazie a conquis l'URSS et exterminé les peuples slaves. Dans le Pacifique, les forces japonaises ont entièrement détruit la flotte navale américaine à Pearl Harbour, puis envahi la côte ouest des États-Unis. Assiégés également à l'est par les forces armées allemandes, les États-Unis doivent se rendre aux deux empires en 1948, provoquant la dislocation du pays. Désormais en guerre froide avec son ancien allié japonais qu'il méprise, le Reich aspire à la colonisation du système solaire pour étendre son empire, après avoir entre autres gagné du terrain sur l'eau en asséchant la Méditerranée. Du fait de cette invasion germano-japonaise, une partie importante de l'histoire des États-Unis n'a pas eu lieu, et notamment la lutte pour les droits civils, si bien que sur la côte ouest américaine, sous contrôle japonais, où se déroule en grande partie l'histoire, les catégories sociales sont fondées sur des distinctions raciales : les noirs sont des esclaves servant les maitres japonais, alors que les blancs, certes mieux lotis que les noirs, sont dans la position du colonisé humilié.

Nous allons voir que les deux civilisations, orientale et occidentale, représentées par les deux puissances qui se partagent le monde, ont un rapport au temps, à l'espace et au langage radicalement différent. Mais elles se rejoignent dans l'idéologie xénophobe qui les sous-tend, portée par une propagande qui a particulièrement bien fonctionné, puisque les personnages n'en perçoivent plus les coutures. Dans une atmosphère de paranoïa généralisée, chaque individu est cantonné dans ses certitudes idéologiques qui l'empêchent d'avoir un «vrai » dialogue avec l'autre. C'est dans l'espace narratorial que les pensées sincères se disent, sous la forme d'un discours indirect libre qui certes permet l'expression libre mais révèle aussi l'impossibilité de l'échange authentique dans The Man in the High Castle.

\section{Contextualisation et décontextualisation}

Si les deux cultures sont toutes deux sous la férule d'un prophète ou d'une voix prophétique, elles diffèrent dans la force performative accordée à ces deux paroles supérieures. Toujours en vie, Hitler, sénile et isolé dans un sanatorium, exerce encore une fascination mystique sur le peuple allemand, 
alors même que l'on sait le dictateur malade et fou ${ }^{2}$ : "The entire world knew it, and yet the leader's gabble was still sacred, still Holy Writ » (Dick 36). Dans la logique allemande, les paroles proférées par les instances au pouvoir sont immédiatement performatives : les Allemands font ce qu'ils disent. Si dire c'est faire en Allemagne, très souvent l'action a le primat sur la réflexion, comme l'énonce Joe, un nazi en service commandé : «Listen, I'm not an intellectualFascism has no need of that. What is wanted is the deed. Theories derive from action» (Dick 161). Certains américains admirent secrètement l'audace et l'efficacité du primat de l'action sur les discours en Allemagne : "And when they did a task they did it right» (Dick 25). Les Japonais sont eux sous l'autorité d'un ouvrage ancestral appelé I Ching, livre de sagesse confucianiste utilisé chez les Taoistes, composé de 64 hexagrammes. Le demandeur obtient des réponses aux questions qu'il se pose en lançant des petites tiges en bois jusqu'à ce qu'elles forment les six lignes de l'hexagramme, puis il consulte l'I Ching pour découvrir à quelle situation cosmique le schéma obtenu correspond $^{3}$. Ainsi l'Américain Frank Fink, converti à l'I Ching, obtient ici l'hexagramme 15, c'est-à-dire «Ch'ien. Modesty. Ah. The low will be raised up, the high brought down, powerful families humbled; he did not have to refer to the text-he knew it by heart. A good omen. The oracle was giving him favorable council» (Dick 13). Fort de ce conseil, il peut dès lors agir en conséquence : «All he could do was adopt the point of view of Hexagram fifteen ». Les paroles de l'oracle guident donc les actions humaines mais leurs effets sont ici perlocutoires: dans l'espace entre l'énoncé et l'effet qu'il produit, se situe la liberté interprétative du demandeur ; car il s'agit de trouver dans le contexte générique des paroles de l'oracle, prenant les contours de l'haïku poétique, les indices d'une situation particulière :

The wall falls back into the moat.

Use no army now.

Make your commands known within your own town

Perseverance brings humiliation (Dick 50)

L'interprète contextualise une pensée décontextualisée, ajustant les paroles invariantes de l'I Ching aux spécificités d'une situation personnelle ${ }^{4}$.

2 Dans la cosmologie nazie, comme l'indique Patricia Warrick, s'appuyant sur les travaux de Jean-Michel Angebert (The Occult and the Third Reich: The Mystical Origins of Nazism and the Search for the Holy Grail, 1971), Hitler fait figure de «prophète apocalyptique » à l'approche du nouveau millénaire (Warrick 176).

3 Un hexagramme est fait de six lignes de Yin (demi ligne) et de Yang (ligne pleine).

4 On retrouve une même forme de présentification et de personnalisation dans la lecture des horoscopes par exemple. Le pronom « your » de l'haïku est à la fois un pronom générique (tout lecteur potentiel) et spécifique (il s'adresse toujours à la personne qui le lit). Les publicités travaillent également sur l'ambiguïté référentielle des pronoms personnels ou des articles indéfinis (cf. Adam et Bonhomme 2007). 
La logique allemande n'entend pas donner un contexte à la parole prophétique : elle aspire au contraire à une décontextualisation. En effet, pour le Reich promis à un avenir supérieur, les choses et les individus de ce basmonde deviennent invisibles : les visées impérialistes allemandes transcendent le monde méprisable des hommes. On envisage pour l'allemand-type, l'archétype aryen, clone d'un modèle invariant, un avenir inter-galactique, afin de hisser son destin au niveau de l'œuvre divine. L'abstraction idéale, sans contingences, que l'esprit allemand cherche à incarner correspondrait, en termes linguistiques, à la «notion» nominale, rompant tout ancrage dans une réalité sociale particulière, comme en témoigne cette citation où les termes assortis de l'article zéro réduisent à néant les spécificités individuelles :

Their view, it is cosmic. Not of a man here, a child there, but an abstraction: race, land. Volk. Land. Blut. Ehre. Not of honorable men but of Ehre itself, honor; the abstract is real, the actual is invisible to them. Die Güte ${ }^{5}$, but no good men, this good man. It is their sense of space and time. They see through the here, the now, into the vast black deep beyond, the unchanging. (Dick 41)

Si les nazis désirent sortir de l'histoire 6 , les japonais vivent bel et bien dans le présent hic et nunc, mais ils l'envisagent comme un processus dynamique en perpétuel état de changement, ne marquant aucun ancrage dans une situation d'énonciation-repère qui définirait un passé ou un futur ${ }^{7}$. C'est alors le présent simple, temps de référence de l'I Ching, qui signifie cette succession de présents sans cesse renouvelés. "The Moment changes» dit le texte (et non «the moment is changing $»)$ : le procès n'est perçu ni comme une occurrence unique, ni comme un énoncé de propriété a-temporel. La forme aspectuelle simple exprime une forme de permanence (du changement), tout en se référant à un contexte toujours spécifique, ce qui l'oppose à la notion (nominale) générique nazie qui se coupe, elle, de toute temporalité (verbale) ${ }^{8}$.

5 La bonté, comme qualité abstraite.

6 Se coupant du hic et nunc, les Allemands sont sortis de l'histoire et donc de la vie sub-lunaire: «Determination to exhalt and immolate themselves so as to obtain a niche in history from which no life form can cast them, no matter what may transpire » (Dick 99).

7 Dans la cosmologie taoiste, les roues de la fortune sont susceptibles de tourner à chaque instant ; c'est le renouvellement permanent qui caractérise les différentes forces en présence : "The Tao is that which first lets the light, then the dark. Occasions the interplay of the two primal forces so that there is always renewal» (p. 106). C'est cet équilibre de la cosmologie harmonieuse de l'I Ching fondée sur la complémentarité des forces de vie et de mort (le yin et le yang) que met en péril le génocide allemand sur terre, comme l'énonce Symons : "To separate yin and yang is to divide the world between necessary destruction and creation, death and life. Paradoxically, the Nazi quest for the 'high castle' of the uniformly white Aryan absolute, culminates in their denial of life [...]. [They] won the war but lost the world » (Symons 266)

8 Outre les frontières spatio-temporelles, ce sont les frontières sacrées du corps que les nazis ont traversées; à l'ère de la reproductibilité technique, au nom de la rentabilité économique, les corps humains de l'Afrique colonisée ont été transformés en objets de consommation courante, opérant une 
La communication entre ces deux empires aux aspirations antinomiques ne peut qu'être problématique. Connaissant l'incapacité nazie de faire dans la poésie (dans tous les sens de l'expression) - l'Allemand est en effet du côté du littéral, de l'univoque -, les messages que les japonais d'Amérique envoient à l'autorité centrale à Tokyo sont sous la forme de messages poétiques codés : «the cipher was the metaphor type, utilizing poetic allusion, which had been adopted to baffle the Reich monitors » (Dick 21). Les rapports entre les deux puissances se réduisent à des échanges cordiaux consistant à ne rien se dire, afin d'éviter tout risque de malentendu. Ainsi, lorsque le responsable japonais Tagomi doit écrire une lettre de condoléances, à la suite de la mort du chancelier allemand, il demande à sa secrétaire de compléter son message : "'Send that' he told her 'Sign it et cetera. Work the sentences, if you wish, so that they will mean something'. As she started from the office he added, 'Or so that they mean nothing. Whichever' » (Dick 90). Il s'agit de faire en sorte que les mots soient vidés de leur force actualisante, pour devenir des coquilles vides sans effets, sans conséquences. Degré zéro de l'échange linguistique, ces rapports diplomatiques garantissent une paix fragile entre les deux nouveaux leaders du monde.

Dans ce monde déboussolé par l'horreur nazie, l'I Ching représente un cadre de référence restaurateur d'harmonie. Cependant, la colonisation japonaise ne se rend pas moins coupable d'une forme de racisme qui ne se différencie de la xénophobie allemande que par son invisibilité.

\section{Xénophobie et propagande}

Philip K. Dick propose ici une illustration fictionnelle de deux formes de « racisation » que la psychosociologie a théorisées. En effet, dans le système national-socialiste allemand, l'identité est fixée par des critères biologiques, inscrits dans le corps : pour les nazis, il n'existe qu' « une seule race qui est le 'nous' » (Sanchez-Mazas 42). Ce processus de biologisation a pour conséquence l'exclusion radicale de ce qui n'est pas «nous » et notamment le juif que l'on déshumanise en lui retirant ses droits, "condition préalable à l'entreprise exterminatrice » (Sanchez-Mazas 44) ${ }^{9}$. Dans The Man in the High Castle, à l'unicité de la race fondée sur des critères de sang répond l'unicité de la langue.

reconversion de l'animé humain en inanimé utile : «Prehistoric man is a sterile white lab coat in some Berlin university lab, experimenting with uses to which other people's skull, skin, ears, fat could be put to. Ja, Herr Doktor. A new use for the big toe; see, one can adapt the joint for a quick-acting cigarette lighter mechanism. Now, if only Herr Krupp can produce it in quantity » (Dick 12). Dans l'esprit japonais en revanche, l'être humain est aussi sacré que les petites choses qui composent l'univers avec lesquelles les hommes sont en communication, voire communion, à chaque instant.

9 Dans le roman, Frank Fink sera arrêté par la police allemande sans passer par la case tribunal. Il ne sera pas entendu puisqu'on ne reconnaît pas aux juifs de droit à l'expression. 
La victoire des allemands a changé la donne en termes d'impérialisme linguistique ; la hiérarchie langagière suit le nouvel ordre des choses : «In German first, then Japanese, and at last English » (Dick 38 $)^{10}$. La logique d'exclusion typique du système nazi s'oppose à la logique caractéristique du colonialisme et de l'esclavagisme qui consiste non pas à exclure l'autre mais à le dominer et à l'exploiter. Dans ce deuxième cas, la race n'est pas localisée dans le nous mais elle est au contraire le fait de ceux qui ne sont pas comme nous : c'est la race de l'autre qui est le signe de son infériorité et qui justifie son exploitation (Sanchez-Mazas 30$)^{11}$. Dans le roman de Dick, la domination japonaise ne repose pas sur l'extermination de l'autre mais sur sa soumission implicite en vertu du pouvoir que la colonisation du pays lui confère. Dans une nation japonaise très attachée au respect des lois, les Américains colonisés ont un statut juridique garanti; mais s'ils sont reconnus comme citoyens, c'est de reconnaissance sociale qu'ils sont privés, puisque les postes les plus hauts placés sont occupés par les colonisateurs. De façon significative, le terme le plus employé tout au long du roman est le signifiant «place » pour indiquer non pas l'espace géographique mais le prestige social : «this stylish high-place young Japanese» (Dick 109). La langue n'échappe pas à ce système de valeurs ; l'utilisation de mots d'origine latine est un indicateur de prestige: « using high place Latin words » (Dick 17).

Chaque individu est donc assigné à une «place » particulière sur l'échelle graduée des valeurs. Si le pouvoir oblige implicitement les citoyens à connaître leur «place », c'est aussi l'esclave qui fait le maître : "It was out of question to let a slave see him [Childan] carrying something. He had to be quite careful of that. A mistake of that kind would cost him dearly; he could never have place of any sort again among those who saw » (Dick 23). Dans The Man in the High Castle, c'est le regard de l'autre qui ordonne et classe les êtres. Il est soustendu par un code linguistique strict, approprié à la condition sociale de l'interlocuteur: "The various modes of address... he knew them. Whom to treat politely, whom rudely. Be brusque with the doorman, elevator operator, receptionist, guide, any janitorial person. Bow to any Japanese, of course, even if it obliged him to bow hundreds of times. But the pinocs ${ }^{12}$. Nebulous area. Bow, but look straight through them as if they did not exist » (Dick 23). C'est à

10 Sentiment de supériorité intériorisé par les Allemands à tel point qu'ils ne peuvent plus comprendre comment la langue allemande peut encore être ignorée par certains : «Clearly the young German could not believe that anyone in the modern world, anyone who had international business dealings and rodecould afford to ride—on the latest Lufthansa rocket, could or could not speak German » (p. 38).

11 L'auteur qualifie d' «autoracisation» la localisation de la race dans le «nous » par opposition à l'« hétéroracisation » qui situe la race dans le « eux » (Sanchez-Mazas 67).

12 Le pinocs est composé de responsables américains ayant un semblant de pouvoir («the puppet white government at Sacramento » [Dick 8]). 
cette invisibilité que Childan tente d'échapper en cherchant à se faire « reconnaître ${ }^{13}$ par les Japonais. À cette fin, il s'efforce de renvoyer au colonisateur l'image que ce dernier se fait de lui ; il s'y conforme jusqu'à l'aliénation ; il singe même l'anglais japonisé de ses supérieurs en supprimant verbes et sujets dans son langage : « how tragic my life » «No. Must investigate». À l'image de l'idéogramme japonais, ses phrases se réduisent à quelques signifiants condensant toute une pensée : « No choice. Idea ». «Difficult matter ». Cet assujettissement linguistique a pour pendant la soumission totale aux idées de l'autre dans le but de lui plaire: "I must follow their leads entirely, he told himself. Agree always» (Dick 108) ou «Was that proper to say? [...] Perhaps that was low-place » (Dick 109).

Mais le mode d'exploitation coloniale est ici compliqué par le fait que les japonais semblent admirer la culture du pays colonisé. Cette soumission au modèle américain transparaît dans le désir obsessionnel des Japonais d'acquérir des objets d'art authentiques appartenant à l'histoire américaine. Ces objets du passé, expropriés de leur contexte, sont devenus des signes extérieurs de richesse. Dans leur propre recherche de reconnaissance sociale, les Japonais pillent ainsi la culture du pays qu'ils colonisent, complexifiant les rapports colonisé/colonisateur, comme le dénonce Childan en les observant : "Witness them drinking from English bone China cups, eating with US silver, listening to Negro style. It's all on the surface. Advantage of wealth and power makes this available to them, but it's ersatz as the day is long » (Dick 112). Cependant, en tant que vendeur d'armes historiques et d'objets d'art américains, Robert Childan sert lui-même ce pillage, ce qui fait de lui, comme le remarque Cassie Carter, un «parasite de parasite » ${ }^{14}$. De même, sa langue, copie d'un anglais japonisé qui est déjà une version appauvrie de l'anglais américain ${ }^{15}$, devient une copie de copie, ce qui contribue à éloigner d'autant l'Amérique de sa propre culture et de sa propre langue.

Pour établir leur domination, les deux empires ont diffusé une propagande sans relâche: du côté allemand, l'inculcation de la supériorité arienne et de la haine du juif est passée par le corps. La réaction épidermique de l'allemand Reiss à la présence de juifs est révélatrice d'un conditionnement

13 Dans le sens d'une reconnaissance sociale telle que la conçoit Paul Ricoeur dans l'opposition actif / passif : «emploi à la voix active - reconnaître quelque chose, des objets, des personnes, soi, un autre, l'un, l'autre - soit à la voix passive - être reconnu, demander à être reconnu » (Ricoeur 37).

14 «But even more significantly, Childan, ever an opportunist, adopts the Japanese taste for American historical objects; that is, he becomes a parasite of the parasites, a mimic of the mimics » (Carter 1995, 336).

15 L'anglais japonais semble plus tenir de la traduction par ordinateur qu'il n'est le fruit d'un apprentissage authentique. Par exemple, le fait d'être «tête en l'air» exprimé en anglais par l'idiome «woolgathering » devient dans la version japonisée : «fleece-seeking cortical response » (Dick 70). On peut aussi citer « nonhabit forming » pour « not addictive » (Dick 72). 
corporel : «And then, all at once, without any warning of any kind, he felt sick with rage. I thought this rage was over, he said to himself. Does it have to go on forever? The war ended years ago » (Dick 129). La pratique japonaise de maîtrise de l'autre est plus invisible, plus implicite : elle consiste, sans violence, à amener l'autre à penser comme soi. La propagande japonaise soumet les êtres en passant inaperçue, comme le confie Childan, victime malgré lui : «Got me to agree, step by step, led me along the garden path to this conclusion [...]. This was how the Japanese ruled, not crudely but with subtlety, ingenuity, timeless cunning. [...] Damn them, I can't free myself of their influence, can't give in to impulse. All spontaneity crushed »(Dick 183). Mais dans le roman, ce n'est pas le processus de lavage de cerveau qui est dépeint, mais les effets d'une propagande réussie. Ce qui était auparavant perçu comme propagande ${ }^{16}$ s'est $^{\prime}$ infiltré dans le discours des personnages : le discours de l'autre (colonisateur ou aryen) est toujours déjà à l'intérieur. Les guillemets du discours citationnel ont disparu, les personnages ont fait leur les idées de l'autre, au point de ne plus percevoir qu'ils les répètent ou qu'ils sont sous influence. La propagande a tellement bien fonctionné que les personnages sont hypnotisés par un système de pensée qui les rend incapables d'échapper aux préjugés qui sous-tendent leur discours ${ }^{17}$. Si Childan, admirateur secret du Reich, veut plaire au japonais, ses paroles sont une citation sans guillemets du discours nazi. L'exemple suivant est une illustration de ce processus d'intériorisation du discours de l'autre ; Childan ne fait que répéter le stéréotype du Polonais voleur alcoolique et stupide qui contraste avec l'image de l'aryen, travailleur propre et assidu: «Those fellows certainly looked happy. And their farms and their cottages were clean. You didn't see pictures of drunken dull-witted Poles any more, slouched on sagging porches or hawking a few sickly turnips at the village markets » (Dick 24). Comme dans tout discours xénophobe, l'autre est construit comme un envahisseur menaçant la nation dans sa souveraineté et l'individu dans son existence ${ }^{18}$. Dans la plus pure rhétorique xénophobe, l'argument de

16 Fink constate que les résistances initiales à l'autorité japonaise lui paraissent aujourd'hui ridicules. La propagande a fonctionné : «In 1947, on Capitulation Day, he had more or less gone bersek. Hating the Japs as he did, he had vowed revenge; he had buried his service weapon ten feet underground, in a basement, well-wrapped and oiled, for the day he and his buddies arose. However, time was the great healer, a fact he had not taken into account. When he thought of the idea now, the great blood bath, the purging of the pinocs and their masters, he felt as if he were reviewing one of those stained yearbooks from his high schooldays, coming upon an account of his boyhood aspirations » (p. 9).

17 Philip K. Dick est peut-être lui-même victime du même aveuglement idéologique : le portrait qu'il dresse du japonais sournois et de l'Allemand rigide repose sur de vieux clichés raciaux. L'uchronie censée bouleverser l'ordre des choses semble verser parfois dans la caricature.

18 Dans la rhétorique xénophobe, l'immigration est la cause principale de phénomènes graves qui «nous menac[ent] de submersion, et à terme de soumission, voire de disparition »(Sanchez-Mazas 201). 
Robert Childan repose ici sur une métaphore conceptuelle ${ }^{19}$ du type IMMIGRATION IS FLOODING associant l'invasion à un cataclysme naturel menaçant de tout emporter sur son passage; il se fait ici le complice de l'idéologie nazie dont les actes d'horreur étaient selon lui nécessaires : "'The world would be much worse. [...] Communism would rule everywhere' [...] 'We have had to suffer, to pay the cost', Robert said. 'But we did it for a good cause. To stop slavic world inundation' » (Dick 111). Cette métaphore idéologique est en mesure de générer une peur panique de l'autre. Reiss compare les juifs à des envahisseurs indestructibles : "That Herr Hope is right, he thought. With his joke about our contact on Mars. Mars populated by Jews. We would see them there, too. Even with their two heads apiece, standing one foot high » (Dick 129). Ce qui commence dans une plaisanterie en vient à prendre corps dans les imaginations, attisant la haine, alimentant un mouvement de «panique » construit de toute pièce ${ }^{20}$.

Le lecteur perçoit dans les discours des personnages les contours des paroles rapportées (sans guillemets). L'enfermement de l'individu dans une idéologie envahissante rend impossible tout échange avec l'autre, qui ne soit pas une confirmation de ses croyances. Le dialogue a pourtant lieu dans l'espace du discours indirect libre mais il ne s'agit là aussi que d'une copie de discours direct.

\section{Le discours dans le discours}

Dans The Man in the High Castle, les êtres ne nourrissent que de la méfiance les uns envers les autres. Les visages sont fermés et les paroles vides, le but étant de ne rien laisser transparaître : "Fortunately, long habit had caused Childan to suppress any show of authentic feelings automatically. $\mathrm{He}$ assumed a bland, sober expression, persona that correctly matched the nature of the situation. He could sense it there, the mask » (Dick 178). Les échanges sont réduits à leur fonction informative. Au téléphone par exemple, on se dispense de la fonction phatique; quand Childan souhaite prendre rendez-vous, il va

19 Nous empruntons bien sûr le terme de métaphore conceptuelle à Lakoff \& Johnson, (Metaphors We Live By, 1980). Voir aussi Andrew Goatly, Washing the Brain. Metaphor and Hidden Ideology (2007).

20 C'est le principe des mouvements de panique morale ou d'hystérie, tels que les a analysés Stanley Cohen dans son livre sur les Mods \& Rockers des années 60. Il montre que la perception de la menace est toujours plus importante que la menace réelle. La réaction de la société à ces violences a fortement contribué à les créer. Or la construction du délinquant comme un envahisseur dangereux par exemple repose sur des analogies fallacieuses : «One of the most misleading and misconceived analogies in regard to explaining delinquency is the attempt to compare it to a disease. People are somehow 'infected' by delinquency which 'spreads' from person to person, so one has to 'cure' the 'disease'. In regard to hooliganism with its distinguishing feature of large public gatherings, this sort of analogy is used even more often and can be propped up with popular versions of mass-hysteria theory » (Cohen 62). 
droit au but: «I wish to see you, private conference, some time today in your office or et cetera » (Dick 58). Lorsqu'il y a échange, on l'a vu, les paroles sont autant de coups linguistiques calculés de façon à obtenir quelque chose ou à s'attirer les faveurs de l'autre dans une stratégie de séduction ou d'humiliation liée à une problématique de pouvoir et d'autorité.

C'est la narration qui offre un espace de liberté expressive aux personnages qui ne peuvent parler ouvertement. La focalisation interne adoptée par le narrateur permet de faire des personnages des «sujets de conscience comme énonciateurs rapportés » pour reprendre les termes de Monique de Mattia-Viviès $(2006,13)$, ce qui leur accorde une forme d'émancipation que les conditions d'échanges en discours réel leur refusent. D'autant que très souvent les pronoms personnels ou les repérages temporels (par rapport aux personnages, sujets de conscience) ne sont pas traduits comme ils devraient l'être dans un récit au passé, provoquant un «effet $»^{21}$ de discours direct. Dans l'exemple suivant, l'alternance du «I $»$ maintenu et du «she » qui marque la présence du narrateur produit une forme de discours indirect libre «hybride» donnant l'impression d'une expression directe du personnage : «I'm sorry, she thought. But she said nothing. I can't save you or anybody else from being dark. She thought of Frank. I wonder if he's dead yet » (Dick 32) ou encore « Joe did not answer. You meant to miss it, Juliana said to herself. I can tell; all at once I know. Then, she thought, you must have been in the war»(Dick 79). L'hybridité du discours rend audible l'expression de paroles impossibles à verbaliser en surface : "Still a little scared of you, she thought. So scared I can't even say it, tell you about it $»$ (Dick 139).

Cependant, ces pensées oralisées se font sur le mode du monologue intérieur où le narrateur rapporte des pensées qui n'ont jamais été verbalisées par le personnage dans le dialogue effectif. La liberté qui semble être accordée au personnage n'est en fait ici, pour suivre les analyses de M. de Mattia-Viviès, qu'un «leurre ${ }^{22}$. Les pensées rapportées forment un « discours sans situation de discours », sans destinataire autre que le personnage lui-même (de MattiaViviès 208) : le conflit reste intérieur et réflexif, dans un mouvement spéculaire destiné à n'avoir aucun effet sur l'autre, puisqu'il ne l'atteint pas. C'est lorsque Childan décide de briser les conventions du code linguistique de la politesse japonaise, de contester l'image que le japonais Paul Karousa se fait de lui, qu'il peut enfin verbaliser ses pensées ouvertement. Refusant en effet de souscrire à

21 «Le paradoxe est l'effet réaliste produit. On entend ces personnages penser alors qu'eux-mêmes ne disent rien. [...] Nous serions davantage ici en présence d'un effet de discours direct libre [...], d'une fiction de DDL » (de Mattia-Viviès 179). J'emprunte également à l'auteur la notion d' « hybridité » du discours rapporté.

22 « Ainsi le MI [Monologue Intérieur] ne serait qu'une fiction de dialogue calquée sur le dialogue effectif, et constituerait ainsi un mode apparemment polyphonique, produisant un effet de polyphonie, sans jamais créer de polyphonie effective » (de Mattia-Viviès 209). 
l'idée du japonais selon laquelle il serait tenté de produire en masse les objets d'art uniques créés par Fink, il exige des excuses de la part de Karousa pour lui avoir prêté ces intentions : « Paul I...am...humiliated [...] and I ask for an apology » (Dick 183). Face à la force illocutoire de cette réplique dont il respecte le courage, Paul s'excuse et pour la première fois baisse les armes de la décence pour exprimer son émotion suscitée par la beauté de l'objet créé par Fink : «I took it upon myself, as I have done with you just now, to deliver an expostulation devoid of tact. This subject carries authority which compels an abandonment of propriety » (Dick 175). Pour créer cet objet d'art, Fink était lui-même allé à l'encontre de l'autorité de l'I Ching dont il n'a pas suivi le conseil et de l'autorité du préjugé intériorisé selon lequel un juif serait incapable de créer, comme le souligne son collègue : «I think you've picked up the Nazi idea that Jews can't create. That they can only imitate and sell » (Dick 49).

Ces nouveaux objets qui semblent posséder selon Karousa une vérité intérieure, appelée $w u$ en japonais, n'exigent de fait aucune validation de la part d'une autorité extérieure. C'est en cela qu'ils font écho plus généralement au travail de l'artiste ${ }^{23}$. L'homme du titre du roman de Dick, The Man in the High Castle, fait référence à un autre écrivain à l'intérieur du livre, Hawthorne Abendsen, lequel imagine dans son roman Grasshopper Lies Heavy ce qui se serait passé si l'axe Allemagne/Japon avait perdu la guerre (autrement dit le monde tel que nous le connaissons). Or à la fin du roman, on apprend que cette version fictionnelle de l'histoire décrit bien la vraie version des faits, provoquant une confusion déconcertante entre réalité et fiction. Il semble que ce brouillage volontaire des frontières soit une réponse à la question posée dans le titre de notre article: si on changeait puis rechangeait le contexte, rien ne changerait. Si l'on changeait les protagonistes sur la scène historique, les mêmes relations de pouvoir et de domination subsisteraient. Childan avait déjà éveillé quelques soupçons en ce sens; on comprend en effet que si les ÉtatsUnis étaient au pouvoir, ils se livreraient à des actes similaires «Think how it would have been had we won! Would have crushed them out of existence. No Japan today and the U.S.A. gleaming great sole power in entire wide World » (Dick 112). Il est difficile de ne pas entendre dans ces paroles une critique implicite de l'impérialisme américain bel et bien au pouvoir dans le vrai monde.

23 C'est l'opinion de John Rieder « It's probably Frank Frink's unthinking production of empty-yet, as we have witnessed, profound and even redemptive-signifiers which stands as the most fitting emblem of the role of the artist in Dick's novel and the political ideology it produces » (Rieder 223). 


\section{Conclusion}

À l'image de l'objet d'art créé par Fink porteur d'une vérité intérieure, il semble que la fiction puisse être porteuse d'une vérité universelle qui transcende tout contexte historique. Mais le roman ne recrée pas moins un contexte de discours qui a son propre destinataire, à savoir le lecteur. De manière certes indirecte, le lecteur pourra toujours apporter une réplique au texte: c'est au final à lui que s'adresse le monologue intérieur des personnages. La fiction opère pourtant une manipulation similaire à la propagande nazie, comme l'expose Reiss rappelant que Goebbels voulait être écrivain. Mais la manipulation fictionnelle sert à dénoncer l'enchaînement des cerveaux par toute forme de propagande. Si le livre de Abendsen est interdit aux États-Unis, c'est parce que la libre circulation de ce texte aurait pour conséquence de laisser s'échapper des effets perlocutoires non maîtrisables. C'est la puissance du regard distancé de l'artiste et le pouvoir dévastateur de ses mots qui exigent, aux yeux du nazi, qu'on le baillonne: "Yes the novelist knows humanity, how worthless they are, ruled by their testicles, swayed by cowardice, selling out every cause because of their greed-all he's got to do is thump on the drum, and there's his response, and he laughing, of course, behind his hand at the effect he gets » (Dick 128). 


\section{Bibliographie}

ADAM Jean-Michel et Marc BonHOMME, 2007. L'Argumentaire publicitaire : rhétorique de l'éloge et de la persuasion, Paris, Armand Colin.

CARTER, Cassie, novembre 1995 (333-342). « The Metacolonization of Dick's The Man in the High Castle: Mimicry, Parasitism, and Americanism in the PSA ». Science Fiction Studies. Vol. 22. No. 3.

CoHEn, Stanley, 1987. Folk Devils and Moral Panics. The Creation of the Mods and Rockers. Oxford, Blackwell.

DICK, Philip K., (1962), 1992. The Man in the High Castle. Londres, Vintage Books.

De MATTIA-VIVIÈs, Monique, 2006. Le Discours indirect libre au risque de la grammaire. Le cas de l'anglais. Aix-en-Provence, Publications de l'Université de Provence.

RICOEUR, Paul, 2004. Parcours de la reconnaissance. Paris, Éditions Stock.

RIEDER, John, juillet 1988 (214-225). « The Metafictive World of The Man in the High Castle: Hermeneutics, Ethics, and Political Ideology». Science Fiction Studies. Vol. 15. No. 2.

SANCHEZ-MAZAS, Margarita, 2004. Racisme et xénophobie. Paris, PUF.

SYMONS, John L., 1985 (261-275). «The Power of Small Things in Philip K. Dick's The Man in the High Castle », Rocky Mountain Review of Language and Literature. Vol. 39.

WARRICK Patricia, juillet 1980 (147-190). «The Encounter of Taoism and Fascism in Philip K. Dick's The Man in the High Castle». Science Fiction Studies. Vol. 7. No. 2. 